Cahiers d'études africaines

198-199-200|2010

50 ans

\title{
Dernière séance
}

Last Show

\section{Emmanuel Terray}

\section{OpenEdition}

Journals

Édition électronique

URL : https://journals.openedition.org/etudesafricaines/16222

DOI : 10.4000/etudesafricaines. 16222

ISSN : 1777-5353

\section{Éditeur}

Éditions de l'EHESS

\section{Édition imprimée}

Date de publication : 20 novembre 2010

Pagination : 529-544

ISBN : 978-2-7132-2252-8

ISSN : 0008-0055

\section{Référence électronique}

Emmanuel Terray, «Dernière séance », Cahiers d'études africaines [En ligne], 198-199-200 | 2010, mis en ligne le 02 janvier 2013, consulté le 23 avril 2022. URL : http://journals.openedition.org/ etudesafricaines/16222; DOI : https://doi.org/10.4000/etudesafricaines.16222

Ce document a été généré automatiquement le 23 avril 2022

(C) Cahiers d'Études africaines 


\title{
Dernière séance
}

\author{
Last Show
}

\section{Emmanuel Terray}

1 Les Cahiers d'Études africaines me font l'honneur et l'amitié de publier l'exposé que j'ai présenté à la fin de l'année universitaire 2000-2001, à l'occasion du dernier séminaire de ma carrière d'enseignant. Qu'ils en soient ici remerciés.

2 C'est aujourd'hui le dernier séminaire de ma carrière d'enseignant, et je voudrais remercier chaleureusement tous les auditeurs qui ont bien voulu venir participer à cette séance. Comme je l'ai annoncé, je souhaite saisir cette occasion pour évoquer mon parcours d'anthropologue. Je me livrerai à cet exercice, non pas pour les plaisirs du souvenir ni pour les charmes de la nostalgie, mais parce que mon récit - comme celui que tous mes collègues pourraient présenter en pareil cas - révélera peut-être quelque chose de nos succès et de nos échecs, de nos illusions, de nos déceptions et de nos doutes; bref il traduira, dans le prisme et les limites d'un itinéraire individuel, une partie au moins de ce qu'a été l'histoire intellectuelle de notre discipline depuis quelques décennies.

\section{Les piliers de l'anthropologie française}

3 Quel paysage offrait l'anthropologie française au moment où les chercheurs de ma génération s'apprêtaient, comme dirait La Marseillaise, à " entrer dans la carrière »? Elle reposait principalement sur trois piliers, que j'appellerai, pour faire bref, le pilier " Griaule », le pilier « Lévi-Strauss » et le pilier « Balandier ».

4 Les anthropologues du premier pilier se singularisaient à nos yeux par un intérêt exclusif porté à la représentation et aux discours, et par leur indifférence à la réalité du social. Sur le plan des sources, cela se traduisait par le privilège accordé à de véritables « auteurs », tel Ogotommêli dans Dieu d'eau, au détriment de la parole commune. Je me souviens d'avoir entendu un jour Germaine Dieterlen remarquer : " Les anthropologues anglais s'intéressent à ce que font les gens, les Français à ce qu'ils disent. » Dans mon for intérieur, je me sentais alors profondément britannique... 
5 En ce qui concerne Claude Lévi-Strauss, j'ai déjà évoqué la double fascination exercée sur moi par Tristes tropiques et par les Structures élémentaires de la parenté1. Fascination affective, pour Tristes tropiques: en dépit de la célèbre première phrase du livre, j'y découvrais le défi du voyage et l'appel de l'ailleurs, l'exaltation de la diversité humaine, la réhabilitation quasi-rousseauiste de la prétendue «sauvagerie », enfin la séduction de ce parcours initiatique que devenait, sous la plume de Lévi-Strauss, l'enquête de terrain. Fascination intellectuelle pour les Structures élémentaires de la parenté: comment ne pas saluer l'étonnante performance scientifique accomplie dans le livre: la réduction du chaos de l'empirie à un ordre intelligible, la multiplicité hétéroclite des systèmes d'alliance ramenée aux trois modalités du mariage entre cousins croisés, échange restreint, échange généralisé, échange restreint différé? Je me souviens d'avoir lu la même année le Capital de Marx, la Science des Rêves de Freud et les Structures élémentaires de la parenté, et d'avoir assisté chaque fois au même exploit : la dissipation de la confusion et l'avènement de l'intelligibilité.

6 Dès cette époque, cependant, l'entreprise de Lévi-Strauss suscite en moi quelques réserves; en premier lieu, si le "paradigme linguistique » semble fonctionner si bien, c'est parce que Lévi-Strauss s'intéresse avant tout à ce qui, dans le social, est discours des terminologies de parenté aux récits mythiques - et tend à ne considérer la pratique que comme l'application d'une règle, sur le modèle du rapport de la langue à la parole. En second lieu, à définir le social par la réciprocité et par l'échange échange des mots, échange des biens, échange des femmes - on laisse échapper les relations unilatérales et dissymétriques, et très précisément la production matérielle et le pouvoir, les deux « portes » par lesquelles l'histoire fait irruption dans notre champ. En troisième lieu, la conception lévi-straussienne de l'intelligibilité pose problème: rendre un donné intelligible, ce serait l'introduire comme variante dans un système de transformations. Celui-ci ne présente cependant qu'une gamme de possibles : qu'en estil du passage au réel ? Pourquoi telle société historiquement déterminée retient-elle telle variante - tel système de parenté, tel ensemble mythologique? Ici le structuralisme reste muet. Plus précisément, ou bien il recourt à la métaphore du choix, ou bien il cède la parole aux historiens. Enfin, le primat de la série paradigmatique - les systèmes de parenté, les mythes - sur la chaîne syntagmatique telle société, conçue comme assemblage de plusieurs segments paradigmatiques appartenant à des séries différentes - ce primat contredit d'une certaine façon l'importance accordée à l'enquête de terrain, car celle-ci s'adresse en priorité aux chaînes syntagmatiques.

7 Bref, le philosophe en moi était fasciné par l'œuvre de Lévi-Strauss mais le militant, passionné d'histoire, de politique et d'action, n'y trouvait pas son compte. D'où mon intérêt pour le troisième pilier, incarné par Georges Balandier. Celui-ci se rapprochait de l'anthropologie britannique du fait d'une commune inspiration durkheimienne, mais il en rejoignait l'aile la plus engagée, la plus sensible aux contradictions, au changement et à l'histoire, à savoir Max Gluckman et l'école dite de Manchester. Son attention se tournait donc avant tout vers les dysfonctionnements et les conflits, regardés à la fois comme "révélateurs du social» et comme moteurs de l'évolution. Balandier s'attachait à l'histoire sous ses formes les plus contemporaines, à travers l'analyse de la situation coloniale et des entreprises de modernisation et de résistance qu'elle suscitait. Enfin, tandis que les structuralistes débutants partaient de préférence pour l'Amazonie ou pour l'Océanie, vers de petites sociétés relativement à l'abri des 
turbulences du monde, les élèves de Georges Balandier se rendaient en Afrique, sur un immense continent en voie de mutation accélérée.

8 J'ai évoqué plus haut le philosophe et le militant. Il faut rappeler qu'à l'époque - le début des années 1960 - il n'y avait guère de formation spécialisée en anthropologie ; les apprentis anthropologues venaient d'ailleurs: de la littérature (Pierre Bonnafé, Marc Augé), de la géographie (Françoise Héritier) mais surtout de la philosophie (M. Izard, Pierre Clastres, Lucien Sebag, Michel Cartry, Alfred Adler, Jean Bazin, et moi). Je ne parle maintenant que pour moi : si j'ai renoncé à la carrière philosophique, c'est par aspiration au réel, au vivant, à la rencontre ; la philosophie, c'était une vie enfermée entre les murs de la salle de cours et ceux de la bibliothèque. Mais je crois n'avoir jamais abandonné les questions de la philosophie; l'anthropologie m'est apparue comme une voie détournée pour penser l'unité de l'esprit et du genre humains, le fondement du social, les logiques à l'œuvre dans l'histoire.

9 De ce point de vue, un changement décisif s'est produit depuis cette date dans notre discipline: elle s'est donné les moyens d'une formation spécialisée. Elle y a certainement beaucoup gagné en rigueur et en précision - il suffit de rappeler sur ce point les tâtonnements et les incertitudes de nos premiers pas - mais elle y a peut-être perdu une partie de son «aura » et de ses horizons. J'ai écrit un jour qu'on pouvait distinguer deux anthropologies (Terray 1988) : celle qui va de l'identité à la différence - qui part des similitudes apparentes pour les faire éclater et mettre en évidence la diversité du social ; celle qui part au contraire de cette diversité pour retrouver ou reconstituer, derrière les apparences multiples, une seule et même espèce humaine. Les deux volets sont complémentaires, et chaque anthropologue les utilise en alternance, mais l'accent peut être mis sur l'un ou sur l'autre. Pour moi, j'étais et je demeure un adepte résolu de la seconde variété; le philosophe rejoint ici le militant: l'unité du genre humain est l'enjeu décisif, du point de vue scientifique comme du point de vue politique.

10 J'étais enfant à l'époque de l'Occupation, assez âgé cependant pour garder le souvenir des étoiles jaunes que portaient certains passants ou même certains camarades de classe ou de jeu. Plus tard, $\mathrm{j}$ 'ai acquis une première conscience politique dans le combat contre le colonialisme: le premier meeting auquel j'ai assisté, en 1953, avec Pierre Stibbe, Yves Dechezelles et Edmond Michelet, était consacré à l'amnistie des condamnés politiques malgaches; ce fut ensuite l'Indochine, la Tunisie, le Maroc, l'Algérie. Or le racisme apparaissait comme l'inséparable compagnon du colonialisme, sa rationalisation, sa justification idéologique; je suis donc devenu alors un défenseur résolu, non seulement de l'unité fondamentale de l'espèce humaine, mais de l'égale dignité des sociétés et des cultures. Les raisonnements tenus par Claude Lévi-Strauss dans Race et histoire - sur le caractère arbitraire des critères utilisés pour hiérarchiser les cultures, sur la loi qui fait naître toute réussite culturelle de la fusion d'apports divers - m'ont semblé et me semblent toujours décisifs, même au prix d'un certain relativisme...

11 De ce fait la pratique de l'anthropologie en tant que telle m'a toujours paru indissociable d'un certain engagement militant, au moins sur des causes de cette nature. D'où la part que j'ai prise en 1986 à la préparation du colloque de l'Association française des anthropologues (AFA) : «Vers une France multiculturelle », à la veille de l'arrivée au ministère de l'Intérieur d'un certain Charles Pasqua; d'où ma fureur de 1996 à la vue du traitement infligé aux sans-papiers. Je pense qu'aujourd'hui ce lien 
entre anthropologie et engagement s'est relâché: ici encore, la discipline y a certainement gagné en "objectivité », en " sérénité ", en " sérieux », en respectabilité académique ; elle y a peut-être perdu un peu de ce qui, selon moi, était son âme...

\section{L'Afrique}

12 Mon premier contact avec l'Afrique, je le prends en 1962-1963 au Sénégal, avec une recherche de caractère sociologique consacrée au syndicalisme. L'année suivante, je gagne la Côte-d'Ivoire, et je m'engage dans une première enquête proprement anthropologique : la rédaction d'une monographie villageoise, réalisée en pays dida. Cette enquête est menée avec des instruments très traditionnels; elle demeure assujettie à deux schèmes classiques. En premier lieu, ce que j'appellerai le tête-à-tête ethnologique : l'enquête regardée comme une rencontre exclusive entre un chercheur et une société, ce qui présuppose que l'unité d'une société globale à ses différents niveaux peut être donnée en une vision synthétique à un chercheur unique. En second lieu, la conception de l'ethnie comme une espèce animale, permettant l'extrapolation du particulier au général et de l'individuel au collectif: à partir de l'examen d'un village, et de quelques coups de sonde aux environs, on croit possible de discourir sur la société dans son ensemble; de ce point de vue, mon travail sur les Dida est manifestement « pré-sperbérien » (Sperber 1975)...

13 Au surplus, mon arsenal conceptuel m'est fourni par une sorte de koiné empruntée au fonctionnalisme britannique. Elle se caractérise par le primat d'un modèle inconscient venu de la biologie; la société est pensée comme un organisme placé dans un milieu. Les institutions sont en conséquence regardées comme des organes, définis par leur fonction, par leur rôle dans la vie du tout. Chaque institution est décrite comme une solution à un problème, qu'il s'agisse de régulation interne ou de rapports avec le milieu. Une pareille vision implique presque inéluctablement l'hypostase de la société en un sujet capable de se poser des questions et de leur inventer des réponses, ce qui exige qu'il soit pourvu de conscience, d'intelligence et de volonté. L'analyse est alors dominée par les catégories d'équilibre à l'intérieur et d'homéostasie avec le milieu extérieur, tous deux assurant la reproduction du tout, elle-même pensée comme une cause finale. Dans cette perspective enfin, contradictions et crises deviennent des dysfonctionnements, mis au compte d'une sorte de pathologie sociale.

Rétrospectivement, il me semble que beaucoup de choses se sont jouées pour moi à partir du malaise que j'ai ressenti durant la réalisation de ce travail en pays dida. En premier lieu, l'unité de la société dida est tout sauf évidente : des frontières incertaines, des origines multiples, des coutumes très variées, pas de pouvoir centralisé, pas d'institution commune, pas de référence spirituelle ou mythique partagée; en fait, l'unité est projetée du dehors, par les peuples voisins et par le colonisateur. D'une certaine manière, je me heurte déjà aux difficultés qui entraîneront vingt ans plus tard la remise en cause du modèle de l'ethnie par mes amis Jean-Loup Amselle, Jean Bazin, Jean-Pierre Dozon et Elikia M'Bokolo (Amselle \& M'Bokolo 1985).

En second lieu, la pertinence du modèle de l'organisme m'est apparue comme tout à fait problématique. Regarder la société comme un sujet n'est qu'un procédé facile pour cacher l'ignorance: on doit, et on peut le plus souvent, identifier qui invente, qui décide, et selon quelles procédures. Par ailleurs, comment voir dans le conflit une maladie, alors que dans de très nombreux cas, il procède du cœur même de la structure 
sociale? En pays dida par exemple, la plupart des accusations de sorcellerie surgissent entre un homme et le frère de son père décédé ; de toute évidence, elles traduisent un antagonisme entre la succession verticale, de père en fils, et la succession horizontale, de frère en frère; mais à son tour cet antagonisme naît d'une opposition entre deux principes: le principe de la transmission patrilinéaire des biens et des rangs, et le principe de l'unité du lignage, par-delà la multiplicité des lignées. En pays dida, ces deux principes sont aussi importants l'un que l'autre, et leur entrechoquement est par conséquent structurel.

Dans mon livre, le modèle de l'organisme est finalement récusé au profit d'un modèle $\mathrm{du}$ jeu, dont l'introduction pose davantage de problèmes qu'elle n'en résout ; il permet en effet d'intégrer la compétition entre les stratégies des acteurs, donc de donner un statut au conflit; en revanche, il ne dit rien de l'origine et de la nature des règles, rien des modalités de leur adoption et de leur transformation. Dans ma réflexion, ce modèle n'a pas duré plus longtemps que le temps de rédiger une conclusion; en effet, mon «paysage intellectuel » s'est alors trouvé bouleversé par l'irruption du marxisme, sous la forme que lui donnaient Louis Althusser et ses élèves.

\section{L'œuvre de Louis Althusser}

17 Ce qu'a signifié pour beaucoup d'entre nous l'œuvre d'Althusser me parait aujourd'hui encore dissimulé par un malentendu profond, dont Althusser porte d'ailleurs lui-même une part de responsabilité. Comment se présentait le marxisme français avant l'entrée en scène d'Althusser? Au sein du Parti communiste français ( $\mathrm{PCF}$ ) régnait un marxisme de type stalinien, mécaniste, évolutionniste et déterministe, dominé par des schèmes comme la théorie du reflet et la succession des stades; le développement des forces productives était posé comme le moteur ultime de l'histoire. Même si des voix dissidentes comme celle d'Henri Lefebvre essayaient de se faire entendre, cette version ossifiée se dressait devant nous comme un monument inébranlable, qu'il fallait accepter ou refuser «en bloc». À l'extérieur du PCF dominait un marxisme "humaniste", qui tenait les œuvres de jeunesse de Marx pour décisives et qui soulignait l'influence de Hegel et le rôle capital de la notion d'aliénation. Les principaux acteurs de ce courant étaient Jean Hyppolite et les Révérends Pères Bigo et Calvez. Les deux versions étaient pour moi inacceptables; la première, sectaire et simpliste, ne permettait pas de paraitre intelligent, ce qui est un obstacle difficilement surmontable pour un intellectuel de vingt-cinq ans; la seconde, plus subtile et plus ouverte, «banalisait » cependant le marxisme et le transformait en une variété particulière de la philosophie de l'existence, ce qui ne convenait pas à mon désir de rupture.

J'étais comme beaucoup, sensible aux efforts de Sartre, dans Questions de méthode et dans la Critique de la raison dialectique, pour rompre avec le mécanisme et l'économisme, pour restituer à la dialectique son rôle. Mais pour Sartre, ces efforts ne pouvaient aboutir que si le marxisme était enraciné dans une philosophie du sujet, entendu comme conscience et comme liberté, et il travaillait donc à cet enracinement. Or je n'acceptais pas une telle perspective : attentif comme je l'étais à la psychanalyse et à la linguistique, la notion du sujet comme foyer ultime du sens ne pouvait que m'inspirer la plus extrême méfiance.

19 L'entreprise althussérienne m'a donné, à moi comme à bien d'autres, le sentiment d'un horizon jusqu'alors bouché qui s'ouvrait, d'une voie obstruée qui soudain se dégageait. 
Deux aspects sont ici particulièrement importants. En 1960, je l'ai dit, le marxisme « officiel » est pétrifié, replié sur lui-même, complètement fermé aux grands courants de la rationalité de l'époque. Althusser va s'attacher au contraire à renouer avec ces courants - avec l'épistémologie de Bachelard et de Canguilhem, avec la psychanalyse de Lacan, avec l'anthropologie structurale de Lévi-Strauss - et il va s'efforcer d'intégrer au marxisme leurs résultats et leur esprit. Mais en introduisant la modernité dans le marxisme, il installe du même coup le marxisme dans la modernité.

Mais surtout les principales catégories développées par Althusser visent toutes à restaurer à la fois le réalisme et la liberté du chercheur. Par exemple Althusser s'en prend résolument à la notion hégélienne de totalité expressive et à la conception "spéculaire » de la vérité, selon lesquelles chaque partie est une image des autres parties et du tout : en fait, chaque partie a sa propre consistance et doit être examinée pour elle-même ; le tout résulte de l'articulation des parties, non de leur identité. Cette polémique met donc un terme à tout "réductionnisme ", à toute théorie du reflet. La description de la société comme «totalité complexe à dominante » va dans le même sens: elle souligne la spécificité irréductible de chaque région du social; celles-ci possèdent leurs propres lois et doivent être étudiées dans leur singularité. Le social global devient dès lors l'association de plusieurs systèmes qui agissent certes les uns sur les autres, mais n'en conservent pas moins leur autonomie.

De même, l'idée de surdétermination permet de ratifier la thèse d'une multiplicité des causes en histoire, et d'envisager tout événement comme une " conjoncture ", c'est-àdire comme la rencontre de plusieurs séries causales, qualitativement distinctes et inscrites dans des temporalités différentes. Enfin, la dissociation entre la détermination et la domination, fondée sur une célèbre note de Marx (1959: 93) dans le Capital, ramène le rôle de l'économie à la détermination de l'instance dominante, c'est-à-dire de la région du social dans laquelle se joue la reproduction du système : la politique à Rome, la religion au Moyen-Âge. Le capitalisme devient ainsi le seul régime où l'économie est à la fois déterminante et dominante, ce qui donne libre champ aux chercheurs travaillant dans des sociétés non capitalistes.

Bref, Althusser crée les conditions du réalisme - chaque région du social peut être désormais scrutée dans sa teneur propre, et non pas comme masque, apparence ou reflet - et celles de la liberté, puisque toutes les singularités peuvent maintenant être accueillies, et qu'aucune limite a priori n'est opposée aux investigations des chercheurs.

En ce qui concerne plus particulièrement l'anthropologie, l'apport décisif est celui d'Étienne Balibar, à travers la redéfinition des notions de mode de production et de formation sociale. La notion de mode de production avait été utilisée jusqu'alors comme un moyen de description et de classification : on se souvient des cinq stades de l'économie énumérés par la préface de la Contribution à une critique de l'économie politique. Le mérite d'Étienne Balibar est d'en avoir fait un instrument d'analyse. À partir des thèses du livre III du Capital, il définit le mode de production comme l'ensemble formé par un rapport de production déterminé et par les conditions de sa reproduction. Le rapport de production est à son tour la relation qui s'établit entre producteurs, non-producteurs et moyens de production, selon le double aspect de la mise en œuvre (qui travaille ?) et du contrôle (qui possède ?). Cette relation détermine les modalités de l'exécution du travail et la répartition du résultat, en particulier son partage entre travail nécessaire et surtravail. Elle détermine aussi la forme du pouvoir qui s'exerce sur les producteurs, selon la formule de Marx (1959, Livre III, t. VIII : 171) : 
« Partout où le producteur contrôle les moyens de production, il faut une force extraéconomique pour le contraindre au surtravail." On le voit, le nombre des modes de production possibles ne peut plus être fixé à l'avance ; selon que le producteur est libre ou non, selon qu'il possède ou non ses moyens de production et selon les formes variées de cette possession, on a autant de modes de production différents.

Dans les conditions de la rareté, le rapport de production est toujours antagonique; l'enjeu du conflit est le volume du surtravail, la répartition du produit et la régulation de l'ensemble du processus. La reproduction est alors la reproduction de cette contradiction; les institutions politiques et les dispositifs idéologiques permettent à la société, non pas de la supprimer, mais de vivre avec elle ; les dominants puisent dans la "superstructure » l'alliage de force et de persuasion grâce auquel ils conservent leur place. Une société concrète se définit enfin comme une formation sociale, comme une combinaison de modes de production dont l'un est dominant, en ce qu'il assujettit les autres aux conditions de sa propre reproduction.

Ici encore, l'apport d'Étienne Balibar m'a paru poser d'un même mouvement les conditions de la rigueur, du réalisme et de la liberté. Rigueur parce que nous disposions désormais d'une définition précise du mode de production, dont nous pouvions identifier les éléments sur le terrain en observant le statut des producteurs, les relations de travail et la distribution du produit. Réalisme et liberté, parce que, je l'ai dit, le nombre des modes de production n'est plus limité a priori; il dépend des résultats de l'enquête. C'est ainsi que nous avons pu enrichir les inventaires existants, en parlant de modes de production cynégétiques (chez les chasseurs-collecteurs), lignagers (dans les sociétés segmentaires) tributaires (lorsque des paysans sont assujettis à des redevances en travail ou en nature), etc. Je reste persuadé, aujourd'hui encore, que, dans le travail des anthropologues, ces instruments n'ont pas été véritablement expérimentés, et que d'une certaine façon, ils ont été abandonnés avant d'avoir servi. Un jour peut être...

Pour ma part, après avoir rédigé à leur propos un texte largement programmatique, j'ai décidé de les mettre à l'épreuve, et c'est une des préoccupations qui m'ont animé lors de mon second travail de terrain, parmi les Abron du nord-est de la Côte-d'Ivoire.

\section{Histoire du royaume abron}

Pour parler de mon "Histoire du royaume abron» (Terray 1995), je dois d'abord me garder des tentations de la cohérence rétrospective, ne pas donner le sentiment que tout était prévu. Si je décide, à la fin de 1966, de me rendre en pays abron, c'est d'abord, pour parler familièrement, que j'en ai assez du lignager et du segmentaire et que je veux « voir autre chose »; or le royaume abron m'offre le spectacle d'un État centralisé, riche d'une longue histoire ; en outre, réserve faite d'un ouvrage superficiel de Tauxier, il constitue un terrain " anthropologiquement vierge ».

Initialement, toutefois, je veux décrire un système politique et contribuer ainsi à l'essor de l'anthropologie politique, qui est alors en pleine expansion. J'ai raconté ailleurs comment ce projet s'est transformé et comment mon travail a finalement produit un ouvrage d'histoire $^{2}$. Sur le terrain, le précédent historique est très vite apparu comme la forme privilégiée, sinon exclusive, de l'explication: chaque institution est "justifiée " par ses origines historiques, par les circonstances de sa création. Par ailleurs, mon renvoi de Côte-d'Ivoire en 1968 et le fait que l'accès du terrain m'a été 
interdit jusqu'en 1975 m'ont obligé bon gré mal gré à un travail d'archives considérable. Du coup, ce qui était envisagé au départ comme une simple introduction historique est devenu un volume de plus de mille pages. Quel bilan tirer aujourd'hui de ce cheminement qui a duré dix-huit ans?

Par rapport à mes antécédents, mon travail en pays abron est innovant sous six aspects différents que je voudrais énumérer rapidement. En premier lieu, j'ai rompu de façon nette avec le «tête-à-tête ethnologique ». En effet, le royaume abron est très vite apparu comme une entité essentiellement frontalière et comme une unité définie par sa place dans un système global. Une entité essentiellement frontalière : le royaume se trouve à la limite de la savane et de la forêt, du portage animal et du portage humain, de la «zone poudre d'or" et de la «zone cauris", du peuplement akan et du peuplement voltaïque, du commerce nigérien et du commerce côtier, de l'empire asante et de l'empire de Kong. Toute l'histoire abron peut être déduite de cette situation frontalière ; le royaume est souvent apparu comme un satellite partagé entre plusieurs attractions, et ses souverains ont été tentés non moins souvent de jouer sur plusieurs tableaux. Par ailleurs, le royaume se caractérise de façon différentielle par le rôle qu'il joue au sein d'un ensemble plus large, où figurent les Européens, les États de la côte, les États de l'hinterland akan et les États mandé de la boucle du Niger ; auprès de leurs partenaires, les Abron trouvent selon les cas des alliés, des rivaux ou des maîtres ; ils sont en mesure de confronter les expériences et les modèles, et d'emprunter à chacun ce qui peut leur être utile. Dès lors, il est impossible de retracer leur histoire sans l'inscrire dans cet environnement; il faut donc sortir des limites de "la » société et se placer au niveau de la région, ce qui suppose un travail collectif et une coopération suivie entre chercheurs.

Deuxième aspect: le rapport à l'histoire. Le travail d'enquête sur l'histoire implique d'emblée une légitimation de la tradition orale comme matériau pour la recherche. Au début des années 1960, cette légitimation est loin d'être acquise : elle se heurte à une coalition hétéroclite entre historiens classiques et anthropologues hypercritiques, selon lesquels les traditions orales ne sont que des discours au présent, utilisant la référence au passé comme arme dans les combats du jour. De mon côté, je ne sousestime pas les déformations infligées à la tradition par les générations successives. Mais je tiens précisément qu'il s'agit de déformations sur une trame initiale que l'on peut tenter de reconstituer. Les royaumes africains ne sont pas des sociétés totalitaires, capables d'imposer un discours unique entièrement inventé. En Afrique, une version fabriquée trouve toujours ses opposants. La critique et la restauration du récit originel sont donc possibles, à quatre conditions. D'abord, il ne faut pas présupposer que les déformations sont inspirées par des considérations d'utilité et d'amour-propre au sens occidental du terme ; les Abron déforment, mais ils le font en fonction de leurs valeurs, dont il faut dresser au préalable l'inventaire. La deuxième condition porte sur le caractère exhaustif de l'enquête, indispensable pour repérer les dissonances, les contradictions et les dissimulations. La troisième condition est de savoir distinguer entre les divers niveaux de la parole et du savoir : histoire populaire, racontée en public à des fins à la fois esthétiques et moralisatrices; histoire réservée à l'élite, dont la diffusion est restreinte, et qui est utilisée comme une sorte de répertoire de leçons et de précédents à l'usage des gouvernants. Enfin, la quatrième condition - pas toujours réalisable - est de disposer d'un instrument extérieur et indépendant de contrôle : les sources écrites. Dans le cas abron, la confrontation des témoignages est possible et se révèle particulièrement fructueuse : les archives des comptoirs européens permettent 
de confirmer tel ou tel épisode rapporté par la tradition, dont on découvre peu à peu la surprenante véracité. Elles conduisent aussi à de nouvelles questions, parce qu'elles introduisent des données absentes de la tradition; il faut se rappeler sur ce point que nos interlocuteurs ne savent pas ce que nous cherchons et ne répondent qu'aux questions qui leur sont posées. Au total, si les quatre conditions que je viens d'énumérer sont remplies - ou dans la mesure où elles le sont - la tradition orale est un matériau utilisable, et l'historien aurait tort de la négliger.

Troisième aspect : le caractère propre du terrain. Dès mon arrivée en pays abron, j'ai perçu la différence avec le pays dida. La société dida est une société relativement égalitaire, et elle a subi tout le poids du passé colonial: en ces premières années de l'indépendance ivoirienne, le «blanc » reste l'objet d'une sorte de déférence méfiante. Rien de tel parmi les Abron: leur royaume est ancien et chargé de gloire ; j'ai pour interlocuteurs les dépositaires de l'histoire, qui sont en général des aristocrates remplis de fierté, sinon de morgue. Entre eux et moi, le rapport est beaucoup plus égal, et je suis souvent contraint de recourir à une très patiente diplomatie. À côté des refus purs et simples, qui ne sont levés qu'au terme d'interminables négociations, se développent des tentatives de manipulation : il est intéressant pour un chef d'obtenir pour telle ou telle version controversée la ratification du chercheur et de son livre. Enfin le problème de la rétribution devient incontournable. La revendication est d'ailleurs légitime : nous dérangeons, nous travaillons dans notre propre intérêt ("Toi, tu vas faire ta thèse... ", m'a-t-on parfois rappelé). Qu'offrons-nous en échange ? La rétribution n'est pas en ellemême un scandale; ce qui importe, c'est d'empêcher qu'un rapport proportionnel s'établisse entre le salaire et le discours. On peut y parvenir dans une certaine mesure en rémunérant de la même façon toutes les informations, quels que soient leur volume et leur qualité. Mais au total, le meilleur remède c'est la longue durée de l'enquête et la multiplicité des retours. Ce qui est collecté dans les premiers mois est en règle générale très sujet à caution; il faut prouver que le chercheur est bien ce qu'il prétend être, ce qui ne se fait pas en un jour ; il faut aussi qu'il se défasse de sa naïveté initiale. Bref, la familiarité et l'accoutumance engendrées par le temps sont le secret du succès.

Quatrième aspect: comme je l'ai indiqué plus haut, une de mes intentions au commencement de l'enquête est de mettre à l'épreuve les concepts fondamentaux du matérialisme historique. Au terme du travail, je peux décrire la "formation sociale" abron comme la combinaison de trois modes de production :

- un mode de production tributaire, dans lequel des communautés paysannes versent des redevances en travail et en nature à une aristocratie guerrière ;

- un mode de production esclavagiste, dans lequel cette aristocratie guerrière utilise des esclaves pour la culture de ses terres, pour l'extraction de l'or et pour le portage ;

- un capitalisme marchand, dans lequel les Dyula assurent les échanges commerciaux du système, en prélevant au passage un profit de monopole.

33 Au sein de cet ensemble, c'est le mode de production esclavagiste qui domine: des paysans, les guerriers exigent une contribution largement symbolique; ils les mobilisent en effet pour la capture des esclaves dans les contrées voisines. J'introduis en outre l'idée de trois logiques gouvernant respectivement l'action des trois classes majeures de la société : pour les paysans, une logique de la sécurité, par laquelle ils privilégient la production des valeurs d'usage et la reproduction simple; pour les commerçants, une logique du profit, conduisant à l'accumulation des valeurs d'échange; enfin, pour les guerriers, une logique de l'ostentation, entraînant une 
accumulation limitée aux seuls biens de prestige. Ainsi, chaque mode de production se voit attribuer un "moteur » propre, ce qui permet de rompre avec l'hypothèse d'une maximisation non spécifiée et avec l'utilitarisme envahissant des économistes.

Cinquième aspect: la réflexion sur l'État. La distinction proposée en 1940 par EvansPritchard et Fortes entre sociétés à État et sociétés sans État a été inlassablement réfutée depuis cette date, ce qui atteste une belle capacité de résistance... Je tiens pour ma part que la question est insoluble en termes d'institutions : il existe une échelle continue des degrés de centralisation, et il est arbitraire de définir des seuils à partir desquels on pourrait parler de chefferies et d'États. En fait, la naissance de l'État suppose d'abord la "fracture sociale", ou plus précisément une déchirure du tissu social. Dans les sociétés segmentaires, on observe certes une hiérarchie fondée sur le sexe, sur l'âge, sur la génération, mais chacune des catégories ainsi disjointes demeure homogène, et d'un groupe à l'autre, les catégories bénéficient du même statut. Dans les sociétés à État, il y a rupture entre dominants et dominés : le plus enfant des dominants est supérieur au plus âgé des dominés. Deux systèmes lignagers peuvent être associés, mais l'un est défini comme supérieur à l'autre.

Par ailleurs, l'État surgit quand apparaissent des relations sociales spécifiques: nommément le contrat et la violence. Dans les sociétés segmentaires, les liens sont fondés sur le sexe, l'âge, la génération, la parenté, la filiation, l'alliance et enfin le voisinage. Avec l'État entrent en scène le contrat et la violence. Le premier organise de préférence les relations au sein du groupe dominant: les fondateurs se prêtent mutuellement serment, passent un pacte avec les maitres du sol, et se constituent des clientèles en échangeant la protection contre le service ; la seconde régit pour sa part, les relations entre dominants et dominés: assujettissement des communautés paysannes, capture des esclaves. À la différence des relations de parenté qui sont toujours décrites comme "naturelles ", le contrat et la violence sont présentés comme des rapports artificiels, produits de l'action humaine et de l'histoire. Bien entendu, dans de nombreux cas, le contrat n'est que le masque de la violence; de même, la tentation est grande de "naturaliser » les liens artificiels et de recourir au langage de la parenté pour évoquer les relations politiques.

Sixième aspect: l'affirmation d'une autonomie du politique dans le contexte de l'Afrique précoloniale. Pendant une longue période, beaucoup d'anthropologues ont contesté la place du politique dans les sociétés africaines, en la rognant, si je puis dire, à la fois par le haut et par le bas, en la réduisant au profit, soit de la religion, soit de la sphère clanique et lignagère. Lorsque des conflits surgissaient, ils étaient uniformément interprétés comme de simples querelles de pouvoir. Mon livre est aussi une réaction contre cette vision réductrice. J'essaie de mettre en évidence, au sein du royaume abron, l'existence de véritables débats politiques, je veux dire des débats ayant des enjeux politiques - concernant l'organisation du royaume et sa politique étrangère - et opposant des factions politiques, recrutées indépendamment des clans et des lignages, et unies autour d'un projet commun. S'il y a une spécificité de la politique africaine, elle tient, non pas aux problèmes et aux acteurs, mais aux procédures de décision : à la procédure du vote - qui dissimule mal une logique de la balance des forces et de l'affrontement - l'Afrique oppose la procédure de la palabre, qui privilégie en toute circonstance le maintien de l'unité du groupe. J'ai évoqué ailleurs les présupposés sur lesquels se fondent l'institution de la palabre et les règles qui gouvernent sa mise en œuvre (Terray 1987-1989). Qu'il me suffise de dire qu'il y a là 
une expérience politique de grande portée, dont nos réflexions contemporaines sur la démocratie pourraient utilement s'inspirer.

\section{Génération oubliée}

Après avoir écrit mon Histoire du royaume abron (Terray 1995), je n'ai plus guère fait œuvre d'anthropologue, et parfois, ce renoncement m'a été gentiment reproché. Sans le nier, je voudrais plaider ici les circonstances atténuantes.

Lors de la soutenance de ma thèse en 1984, Michel Izard, membre du jury, me déclara au terme d'une intervention très amicale: "Tu es l'ultime survivant d'une époque révolue.» Il me signifiait par là que mes problèmes, mes concepts, mon langage n'étaient plus ceux de l'anthropologie contemporaine - et il n'avait pas tort.

Si j'ai abandonné l'anthropologie, c'est aussi parce que l'anthropologie m'a d'une certaine manière abandonné. La vague des années 1960, qui m'avait porté avec bien d'autres, s'est retirée et m'a laissé sur le sable...

Considérons d'abord l'anthropologie dite " exotique »: depuis vingt ans, elle est très largement revenue à ses objets traditionnels : la parenté, la religion, la mythologie, le rituel. Les champs que notre génération avait privilégiés - l'économie, le travail, les échanges, la politique, l'histoire - ont été désertés par les anthropologues; plus précisément, ils ont été restitués aux disciplines qui les étudient dans les sociétés industrialisées; ce sont des économistes qui examinent désormais les questions du développement, des politologues qui comparent les régimes et les formes de pouvoir, des historiens qui écrivent l'histoire de l'Afrique ou celle de l'Amérique indienne.

41 Cette évolution est, à bien des égards, positive, notamment parce qu'elle remet en cause le partage entre " primitifs » et civilisés qui avait marqué les sciences sociales au $\mathrm{XIX}^{\mathrm{e}}$ siècle, les "primitifs » étant naturellement laissés aux anthropologues... Au surplus, elle est imposée par "la force des choses». Dans le monde réel aussi, les cloisonnements tombent, les discontinuités s'estompent. Sous la double pression de l'hégémonie occidentale et du marché mondial, il se produit une uniformisation progressive des sociétés, qui rend peu à peu caduque l'idée selon laquelle certaines zones devraient se voir appliquer un traitement particulier.

Mais pour notre discipline, la mutation en cours se paie d'un prix relativement élevé : elle implique en effet le renoncement à cette ambition totalisante qui a fait la grandeur et les chefs-d'œuvre de l'anthropologie entre 1920 et 1960. De Malinowski à Fortes, de Nadel à Leach, les anthropologues nous proposaient alors une vision globale, synthétique des sociétés qu'ils étudiaient; du même coup, l'articulation des diverses régions du social, les faits de surdétermination, les dispositifs de causalités réciproques, devenaient pour eux des problèmes majeurs. Leurs entreprises connaissaient des fortunes diverses, elles aboutissaient à des résultats plus ou moins convaincants, mais toutes présupposaient qu'il était possible, non seulement de penser le social, mais de le penser comme un tout. Or, c'est précisément cette conviction qui s'est aujourd'hui perdue.

43 J'ai longtemps pensé que cette difficulté était liée à l'absence d'un modèle, d'un paradigme organisateur. Après tout, l'histoire de l'anthropologie peut aussi être déchiffrée à travers la succession des modèles qu'elle a empruntés à des disciplines voisines pour penser ses objets: modèle évolutionniste issu du darwinisme avec 
Morgan, modèle biologique de l'organisme avec le fonctionnalisme britannique, modèle linguistique avec Lévi-Strauss et le structuralisme. Au moyen de l'anthropologie marxiste, nous avions, nous aussi, tenté d'ajouter un nouveau terme à la série.

À mon sens, notre échec ne s'explique pas seulement par les défauts de notre modèle, et par notre propre insuffisance: comme je l'ai dit, nos propositions n'ont pas été véritablement soumises à l'épreuve de l'expérience. L'échec tient plus profondément au fait que, renonçant à penser le social comme un tout, les anthropologues n'éprouvaient plus le besoin de tels modèles. Contrairement aux apparences, ce n'est pas l'absence de modèle qui provoque le renoncement, c'est le renoncement qui rend tout modèle inutile. Le rôle du modèle est de fournir une « image-guide » du tout : si l'on ne cherche plus à penser le tout, on n'a plus besoin de modèles.

D'où l'étonnant éclectisme dont l'anthropologie actuelle fait preuve dans le choix de ses références théoriques. Cet éclectisme ne présente aucun inconvénient dès lors qu'on abandonne le point de vue de la totalité. Pour saisir le tout, il faut un cadre cohérent, un système de concepts unitaire et consistant, mais quand on ne s'intéresse qu'à des fragments particuliers, considérés isolément, on peut recourir à des instruments incompatibles entre eux, puisqu'on n'est jamais obligé de les utiliser ensemble.

$\mathrm{Au}$ reste, cette situation n'est pas propre à l'anthropologie. De l'avis de nombreux critiques littéraires, il est désormais impossible d'écrire de grands romans comparables à ceux du XIX ${ }^{e}$ siècle, faute d'une vision globale du monde. De son côté, Jean-François Lyotard a souligné la fin des "grands récits " philosophiques. Quant aux économistes, ils ne décrivent la réalité qu'au prix d'hypothèses simplificatrices reléguant dans l'inessentiel des pans entiers de la vie sociale.

Reste à évoquer « l'anthropologie du proche ", qui réunit à présent plus de la moitié des anthropologues. À mon sens, cette anthropologie du proche éprouve de grandes difficultés à énoncer sa spécificité par rapport aux disciplines voisines, en particulier la sociologie. Puisque la frontière ne passe pas par les objets de recherche, la séparation doit tenir au regard, à l'approche, à la méthode. Il n'est guère original de définir l'anthropologie comme ce qui est produit par une enquête de type anthropologique, quelle que soit la latitude sous laquelle cette enquête est réalisée. En d'autres termes, c'est le travail de terrain qui serait le critère de l'appartenance de telle ou telle étude au domaine de l'anthropologie. Encore faut-il que, du travail de terrain comme nous l'entendons, nous puissions fournir une image assez précise pour être discriminante, pour qu'on puisse tracer à partir d'elle des lignes de démarcation nettes et sans ambiguïtés.

Telle qu'elle était conçue et pratiquée jusqu'aux années 1960, l'enquête de terrain présentait plusieurs caractères :

- elle supposait une confrontation approfondie avec l'altérité sociale, culturelle, linguistique, ce qui rendait donc nécessaire une immersion de longue durée dans un milieu étranger au chercheur;

- elle exigeait que soit choisie comme objet de l'enquête une communauté suffisamment restreinte, cohérente et autonome pour contenir en elle-même la plupart des facteurs déterminant sa vie et son destin : une bande, un village, un quartier urbain. Le sens de cette exigence était de faire en sorte que l'essentiel des résultats de la recherche provienne de l'enquête elle-même, et non de sources latérales ;

- elle demandait que l'observation soit privilégiée par rapport à l'entretien, et que l'interférence du chercheur soit réduite au minimum ; 
- elle obligeait la recherche et le chercheur à se soumettre à l'épreuve du temps. Indépendamment de sa productivité, le travail devait se poursuivre pendant de longues périodes continues, et multiplier les retours ;

- enfin tout ceci entraînait le primat du qualitatif sur le quantitatif, de l'intensif sur l'extensif, etc. de toute manière, ne reviendra plus ? L'anthropologie que j'ai connue et pratiquée a eu ses moments de splendeur et ses grands personnages, mais elle a disparu, et ce qui lui a succédé sous le même nom d'anthropologie est en fait une autre activité. Il est donc bien vain de prétendre maintenir artificiellement en vie un corps qui a rendu son dernier souffle. Après tout, le théâtre grec et la musique classique ont vécu des destins semblables : après avoir brillé d'un éclat incomparable, ils sont aujourd'hui des astres éteints. De même, les anthropologues de la vieille école ne forment plus à présent qu'une "armée morte »: il me plaît de penser que j'aurai été l'un de ses derniers combattants. 


\section{BIBLIOGRAPHIE}

AMSELle, J.-L. \& м'BокоLо, Е. (dir.), 1985 Au cour de l'ethnie. Ethnies, tribalisme et État en Afrique, Paris, la Découverte.

MARX, K., 1959 Le Capital, traduction de Joseph Roy, Paris, Éditions Sociales.

SPERBER, D., 1975 Le savoir des anthropologues, Paris, Hermann.

TERRAY, E., 1987-1989 « Un anthropologue africaniste devant la cité grecque », Opus, Rivista internazionale per la storia economica e sociale dell'antichità, VI-VII : 13-25.

-, 1988 Lettres à la Fugitive, Paris, Odile Jacob.

-, 19881995 Une histoire du royaume abron du Gyaman : des origines à la conquête coloniale, Paris, Karthala.

TERRAY, E. \& COLLEYN, J.-P., 2005 Traversées, Bruxelles, Labor.

\section{NOTES}

1. Exposé au séminaire de Michel Izard (1995). Repris dans Emmanuel TERRAY \& JeanPaul COLLEYN (2005: 75)

2. Voir note 1.

\section{RÉSUMÉS}

Résumé

Après avoir dressé un «état des lieux » de l'anthropologie française au début des années 1960, l'auteur évoque les principales étapes de son parcours de chercheur : son travail en pays dida, puis en pays abron. Il rappelle quelles étaient alors les orientations dominantes: histoire, économie, politique, et quels furent à ses yeux les principaux acquis de la période. Il s'interroge pour conclure sur la situation actuelle de la discipline.

Abstract

After drawing up an "inventory" of French anthropology in the early 1960s, the author looks at the main stages of his career as a researcher and his work among the Dida and the Abron. He looks back at the main historical, economic and political trends, and what he considers to be the main lessons of that period. He concludes by reflecting on the present situation of anthropology. 
INDEX

Mots-clés : Pays abron, pays dida, Louis Althusser, anthropologie française

Keywords : Abron Speaking Area, Dida Speaking Area, Louis Althusser, French Anthropology

\section{AUTEUR}

\section{EMMANUEL TERRAY}

École des hautes études en sciences sociales, Paris. 